\title{
Unsaturated fatty acids are the active molecules of a glucan-synthase-inhibitory fraction isolated from entomophthoralean protoplasts
}

\author{
Joanna Mackichan, ${ }^{1,2}$ Lene Thomsen, ${ }^{3}$ James Kerwin, ${ }^{2}$ Jean-Paul Latgé \\ and Anne Beauvais ${ }^{1}$
}

Author for correspondence: Anne Beauvais. Tel: +331456162 25. Fax: +33145688420 .

\footnotetext{
1 Unité de Mycologie, Institut Pasteur, 25 rue du Dr Roux, 75724 Paris Cedex 15 , France

2 Department of Botany, University of Washington, KB-15, Seattle, WA 98195 , USA

${ }^{3}$ Department of Ecology and Molecular Biology, Royal Veterinary and Agricultural University, DK-1870 Frederiksberg C, Copenhagen, Denmark
}

\begin{abstract}
A few entomophthoralean species are able to multiply in a protoplast form. The polysaccharide synthases which synthesize the cell wall are inactivated in this form. An inhibitor of one of the key enzymes of wall synthesis, glucan synthase, was isolated from entomophthoralean protoplasts, using silica column chromatography and HPLC. Thin-layer and gas chromatography revealed free fatty acids in the inhibitory fractions. These fatty acids, including long-chain unsaturated fatty acids, were shown to be responsible for the inhibition of glucan synthase. The fatty acids were generated during incubation of a protoplast homogenate for $36 \mathrm{~h}$ at $37^{\circ} \mathrm{C}$ and were shown to be non-competitive and non-specific inhibitors of glucan synthase.
\end{abstract}

Keywords: glucan synthase, Entomophthorales, Aspergillus fumigatus, fatty acid, 1,3- $\beta$ glucan

\section{INTRODUCTION}

Entomophthorales are entomopathogenic fungi. Some species, such as Entomophtbora schizophorae, Entomophthora muscae and Entomophaga aulicae, have the ability to develop and multiply in vivo as protoplasts, which lack a cell wall. These protoplasts, unlike hyphal bodies, are not recognized by the immune system of insects (Beauvais $e t$ al., 1989). 1,3- $\beta$-Glucan and chitin are present in the hyphal wall but absent on the protoplast surface. These wall components are responsible for the recognition of the mycelium by the immune system, and their absence accounts for the ability of protoplasts to escape encapsulation (Beauvais et al., 1989). Protoplasts and the corresponding walled form, the hyphal bodies, have been cultivated in vitro for Entomopbthora muscae and Entomophaga aulicae. Only the protoplast stage has been cultivated in vitro for Entomophthora schizophorae (Beauvais \& Latgé, 1988).

The major components of fungal cell walls, 1,3- $\beta$-linked glucan and chitin, are synthesized by the glycosyltransferases 1,3- $\beta$-glucan synthase (GS) and chitin synthase, which catalyse the transfer of glucose or $N$ acetylglucosamine units from UDP-Glc (uridine diphosphate glucose) or UDP- $N$-acetylglucosamine into growing polysaccharide chains (Cabib et al., 1988). The level of control which prevents the biosynthesis of cell wall in the protoplasts is not understood, although the membrane-bound polysaccharide synthases, which synthesize the cell wall, are somehow inactivated (Beauvais \& Latgé, 1989). Regulatory mechanisms could operate at the transcriptional or at the translational level, or could involve a specific inhibitor only active in the protoplast stage. The presence of an mRNA specific for one of the chitin synthases found in this species suggested that the transcription of the polysaccharide synthases in the entomophthoralean protoplasts is not blocked (Thomsen \& Beauvais, 1995). However, the transcription of the GS gene or the translation of the mRNAs of both polysaccharide synthases could not be studied, as no gene has been cloned for GS and these enzymes have not been purified. The presence of non-competitive inhibitors of polysaccharide synthases of natural origin has been detected in many fungi (Fèvre, 1979; Iwata et al., 1982; Kang et al., 1986; Sawistowska-Shröder et al., 1984), including the protoplasts of two entomophthoralean species (Beauvais \& Latgé, 1989). 
In this study, an inhibitor of GS was isolated from entomophthoralean protoplasts and then identified in order to evaluate its specificity to the GS and its presence only in the protoplast form. Its activity was tested on the GS of Aspergillus fumigatus, a fungal pathogen of immunocompromised patients. The low sensitivity or resistance of $A$. fumigatus to many antifungal compounds made it a good model for our study (Fromtling \& Abruzzo, 1989; Beaulieu et al., 1993).

\section{METHODS}

Purification of the inhibitor from Entomophthoralean species. Protoplasts were grown in 11 GLEN medium $(0.4 \%$ glucose, $0.5 \%$ yeast extract, $0.65 \%$ lactalbumin hydrolysate, $0.77 \% \mathrm{NaCl}$; Beauvais \& Latgé, 1988), in a cell fermenter (Biolafitte) inoculated with $120 \mathrm{ml}$ protoplasts of Entomophthora schizophorae, Entomophthora muscae (isolates Em2 and $44 \mathrm{E}$ respectively, provided by Dr J. Eilenberg, Royal Veterinary and Agricultural University, Denmark) or Entomophaga aulicae (isolate 2896, provided by Dr D. Boucias, University of Florida, USA). The protoplasts were grown at $25^{\circ} \mathrm{C}$ for $3 \mathrm{~d}$ at 50 r.p.m. and an aeration rate of 0.21 air $\mathrm{min}^{-1}$ outlet located.

Protoplasts were recovered by centrifugation and washed in $10 \mathrm{mM}$ phosphate buffer $(\mathrm{pH} 7 \cdot 8)+1 \mathrm{M}$ sucrose. After breakage in a Potter piston homogenizer in TE buffer $(50 \mathrm{mM}$ Tris $/ \mathrm{HCl}$, $1 \mathrm{mM}$ EDTA, $\mathrm{pH} 7 \cdot 6$ ), the protoplast suspension was incubated for $36 \mathrm{~h}$ at $37^{\circ} \mathrm{C}$ and lyophilized to yield the crude inhibitor fraction. Since the inhibitory fraction was insensitive to pronase or trypsin $\left(1-10 \mu \mathrm{g} \mathrm{ml}^{-1}\right.$ for $4-36 \mathrm{~h}$ at $\left.37^{\circ} \mathrm{C}\right)$, a lipidic structure was expected. A $100 \mathrm{mg}$ sample of the crude inhibitor fraction was extracted (15 min vigorous shaking on a vortex) twice with $25 \mathrm{ml}$ chloroform/methanol $(2: 1, \mathrm{v} / \mathrm{v})$ at room temperature. The extract was then filtered through filter paper to eliminate particulates, and the sample concentrated under vacuum and finally dried under $\mathrm{N}_{2}$. The product was resuspended in $500 \mu \mathrm{l}$ chloroform $\left(\mathrm{CHCl}_{3}\right.$ fraction $)$ and $10 \mu \mathrm{l}$ was used for the $\mathrm{GS}$ assay test.

The $\mathrm{CHCl}_{3}$ fraction was fractionated on a $3 \mathrm{ml}$ Shandon Hypersep silica column for solid phase extraction, with $100 \mu \mathrm{l}$ applied at a time. Columns were eluted sequentially with 1 column volume of hexane, 1 volume of chloroform, and 1 volume of $4: 1(\mathrm{v} / \mathrm{v})$ chloroform/methanol. The $4: 1$ chloroform/methanol fraction, which was found to contain the inhibitor, was then concentrated and resuspended in $500 \mu$ lethyl acetate. Ten microlitres of this $4: 1$ chloroform/methanol fraction was used to test the inhibitory activity.

The $4: 1$ chloroform/methanol fraction was used for HPLC on a Lichrosorb Si $5 \mu 250 \times 4.6$ column, using an isocratic system with hexane/chloroform/methanol $(5: 10: 2$, by vol.), at a flowrate of $1 \mathrm{ml} \mathrm{min}^{-1}$. The run time was $30 \mathrm{~min}$. Before injection, the solvent was totally evaporated and the sample resuspended in the running solvent. A peak of absorbance was observed between 3 and 6 min after injection. A major peak that eluted between 4 and 6 min (collected in tubes 8-12 and termed the 8-12 fraction) contained the inhibitory activity. Aliquots $(1 \mathrm{ml})$ of this peak were pooled, dried down, and resuspended in $200 \mu$ l ethyl acetate. Ten microlitres of each fraction was used to test the inhibitory activity.

The purity of the inhibitory fractions was assayed by TLC. Samples were spotted on Kieselgel $60 \mathrm{~F}_{254} \mathrm{DC}$-alufoil silica plates (Merck) and the plates were run in hexane/diethyl ether/acetic acid $(70: 30: 1)$. Fatty acid (FA) and diglyceride (DG) standards from Sigma were run for comparison. All plates were developed under $\mathrm{I}_{2}$ vapour (Higgings, 1987).

Test of inhibitory activity on GS. To test the inhibitory activity of a fraction, solvent was evaporated under $\mathrm{N}_{2}$ and the sample resuspended in TE buffer. The effects of various standards on GS activity were also assayed in TE buffer. Ten micrograms of DG standards (Sigma), containing equal amounts of dipalmitin, distearin and diolein, were added directly to the GS assay reaction mixture, giving a final concentration of $0.33 \mathrm{~g} \mathrm{l}^{-1}$. Free FAs were assayed, also with a concentration of $0.33 \mathrm{~g} \mathrm{l}^{-1}$. Both saturated $(14: 0,16: 0$ and $18: 0)$ and unsaturated $(18: 1,18: 2$, $18: 3$ and 18:4) FAs were tested. The unsaturated long-chain FAs were also tested at lower concentrations, from 0.33 to $33 \mathrm{mg} \mathrm{l}^{-1}$, using the following GS assay. A solubilized GS extract (P30) was isolated from Aspergillus fumigatus as described by Beauvais et al. (1993). For Trichopbyton rubrum and $T$. mentagropbytes, the high-speed membrane extract (before solubilization) was used as a source of GS. The GS of S. cerevisiae was also used to test the inhibitor (E. Cabib, personal communication).

GS activity was estimated by measuring the incorporation of UDP- $\left[\mathrm{U}_{-}{ }^{14} \mathrm{C}\right]$ glucose into insoluble glucan. To test the inhibitory activity of a fraction, the sample was resuspended in $10 \mu \mathrm{l}$ TE. It was then incubated for $20 \mathrm{~min}$ with $15 \mu \mathrm{l} \mathrm{P} 30$ at room temperature before $5 \mu$ l of the reaction mixture, containing TE $+0.2 \mathrm{M} \mathrm{NaF}+1 \mathrm{M}$ sucrose $+2.5 \mathrm{mM}$ UDP-glucose (with $2 \cdot 4 \mu \mathrm{M}$ UDP-[U- ${ }^{14} \mathrm{C}$ ]glucose, specific activity $293 \mathrm{nCi}$ $\mathrm{nmol}^{-1}, 108 \mathrm{~Bq} \mathrm{nmol}^{-1}$ ), was added. The enzyme reaction was allowed to take place for $45 \mathrm{~min}$, after which the reaction product was precipitated with $50 \mu$ l ethanol and recovered by filtration on a GF/C glass-fibre filter. The filters were dried for $15 \mathrm{~min}$ at $80^{\circ} \mathrm{C}$, washed with $50 \mathrm{ml} 60 \%$ (v/v) ethanol, and then incubated in $2 \mathrm{ml} \mathrm{BCS}$ scintillation fluid for $1 \mathrm{~h}$. The radioactivity was measured in an LKB liquid scintillation counter. Percentage inhibition was measured as the ${ }^{14} \mathrm{C}$ incorporated into insoluble glucan compared to the control without inhibitor. The specificity of the inhibitory activity (fraction 8-12) on GS was tested by incubating this fraction with a chitin synthase extract of $A$. fumigatus obtained as previously described (Beauvais \& Latgé, 1989).

Kinetic analysis of Entomophthora schizophorae inhibitor on the GS. For $K_{\mathrm{m}}$ determination, different concentrations $(0.34-2 \mathrm{mM})$ of the substrate UDP-glucose were used in the reaction mixture, and the reaction was only allowed to take place for $10 \mathrm{~min}$. Since the insolubility of the inhibitor in the reaction mixture did not affect the linearity of the dose-response curve, $K_{1}$ assays were done: the inhibitor concentrations were expressed in terms of c.p.m. of ${ }^{14} \mathrm{C}$ added to the reaction since the molarity was not known. For this purpose, the protoplasts were grown in GLEN medium supplemented with $0.13 \mu \mathrm{M} \mathrm{D}-$ $\left[\mathrm{U}-{ }^{14} \mathrm{C}\right]$ glucose for $3 \mathrm{~d}$. The inhibitor was then purified as described above, and recovered in fraction 8-12. Concentrations corresponding to 225 and 300 c.p.m. were added for competition experiments. For all the combinations of inhibitor and substrate concentration $1 / V$ was plotted against $1 / S$.

General lipase test on Entomophthora schizophorae inhibitor. The inhibitory fractions were treated with a lipase from Candida cylindracea (Sigma) dissolved in TE to a concentration of $0.2 \mathrm{mg} \mathrm{m}^{-1}$, i.e. 12000 enzyme units $\mathrm{ml}^{-1}$. Fractions tested included $\mathrm{CHCl}_{3}$ fraction, the $4: 1$ chloroform/ methanol fraction, and fractions of the HPLC peak eluting between 4 and $6 \mathrm{~min}$. Approximately $0.05 \mathrm{mg}$ of each fraction 
was combined with $0.5 \mathrm{ml}$ of the enzyme/buffer mixture, then incubated for $1.5 \mathrm{~h}$ at $37^{\circ} \mathrm{C}$. Controls were also run with a DG standard (Sigma), consisting of equal amounts of dipalmitin, distearin and diolein, as well as with an enzyme preparation boiled for $10 \mathrm{~min}$. Reactions were stopped by adding $1 \mathrm{ml}$ chloroform to the reaction mixture and the reaction products were extracted by partitioning between the $\mathrm{CHCl}_{3} / \mathrm{H}_{2} \mathrm{O}$ fractions. The chloroform fraction was then concentrated and resuspended in $500 \mu \mathrm{l}$ ethyl acetate. The reaction products were assayed by using $42 \mu \mathrm{l}$ for a GS assay and $10 \mu \mathrm{l}$ for a TLC plate.

Effect of bovine serum albumin on inhibitor and GS activity. Bovine serum albumin (BSA) is known to bind free FAs (Hamilton et al., 1991). The protoplast inhibitor $\left(10 \mu \mathrm{C} \mathrm{CHCl}_{3}\right.$ fraction) and $0.007 \mathrm{mg}$ standard oleic acid (18:1) were combined with varying concentrations of BSA $\left(1.6-8 \mathrm{~g} \mathrm{l}^{-1}\right)$ and added to the GS assay. Residual effects of BSA on GS were tested by running controls with the same concentrations of BSA, without inhibitor. Percentage inhibition was then calculated by comparison with a control with neither inhibitor nor BSA, and plotted against concentration of BSA.

Electrospray ionization of Entomophthora schizophorae inhibitor. After HPLC purification, samples were analysed by electrospray ionization MS on a triple-quadrupole Sciex API III instrument (PE/SCIEX; Kerwin et al, 1994). Samples were infused into the electrospray source via a $50 \mu \mathrm{m}$ i.d. fused silica transfer line using a Harvard Apparatus pump at $3 \mu \mathrm{min}^{-1}$. Positive-ion MS or MS/MS was run with an orifice voltage of $70 \mathrm{~V}$, and the corresponding negative-ion spectra were run at $70 \mathrm{~V}$. The interface temperature was maintained at $52^{\circ} \mathrm{C}$. For tandem mass spectrometry (MS/MS), precursor ions were selected with the first of three quadrupoles (Q1) for collisioninduced dissociation with argon in the second quadrupole (Q2). The third quadrupole (Q3) was scanned with a mass step of $0 \cdot 20 \mathrm{Da}$ and $1 \mathrm{~ms}$ per step. Parent ion transmission was maximized by reducing the resolution of Q1 to transmit a 2-3 $m / z$ window about the selected parent ion, and Q3 resolution was adjusted to approximately $50 \%$ valley between peaks $3 \mathrm{Da}$ apart. Spectra were collected and analysed using software from Sciex Corporation.

GC and GC-MS of fatty acid methyl esters (FAME). Free FAs in the HPLC fractions were methylated using ethereal diazomethane (Kates, 1986). FAME were analysed using a Hewlett Packard 5890 series II gas chromatograph. Using helium as a carrier gas at $2 \mathrm{ml} \mathrm{min} \mathrm{m}^{-1}$, samples were injected (splitless injection) onto a $30 \mathrm{~m} \mathrm{DB}-23$ column (J\&W Scientific). The initial temperature of $120^{\circ} \mathrm{C}$ was held for $2 \mathrm{~min}$ and raised at $4{ }^{\circ} \mathrm{C} \mathrm{min} \mathrm{m}^{-1}$ up to $240^{\circ} \mathrm{C}$ and held for $10 \mathrm{~min}$. FAs were identified and quantified using retention times and GC-MS on a Kratos mass spectrometer.

\section{RESULTS}

\section{Purification of the Entomophthora schizophorae inhibitor}

The results are summarized in Fig. 1. The active fraction was first found in the $\mathrm{CHCl}_{3}$ fraction, then in the $4: 1$ chloroform/methanol fraction, and finally in tubes 8-12 eluted after 3-6 min during the HPLC run. TLC on silica plates revealed at least three different products in the fraction from tubes $8-9$. The spots corresponded roughly to those of standards of DGs and free FAs. The nonactive 6-7 fraction consisted mainly of DGs, while the active 10-12 fraction consisted mainly of FAs. Without the $36 \mathrm{~h}$ incubation period at $37^{\circ} \mathrm{C}$ of the protoplasts,

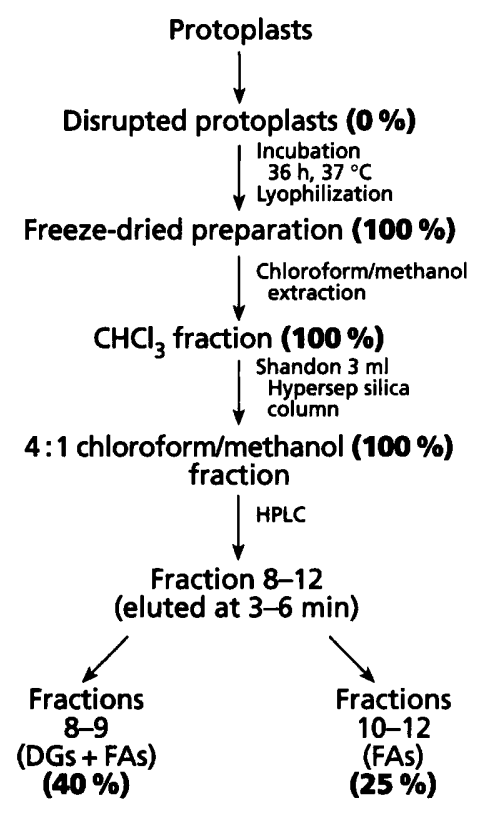

Fig 1. Purification of the GS inhibitor from protoplasts of Entomophthora schizophorae. The percentage inhibition of GS caused by the various fractions is shown in parentheses (bold type); see Methods for details.

there was no inhibitory activity and there was no TLC spot for FAs (data not shown).

The inhibitory fraction was found to correspond to a TLC spot with an $R_{F}$ value of $0.36-0.40$, similar to the area to which unsaturated FAs migrated. Kinetic studies showed that the apparent $K_{\mathrm{m}}$ for UDP-glucose in the standard enzyme preparation, determined by using MichaelisMenten plots, was $1 \mathrm{mM}$. The inhibitor is a noncompetitive inhibitor of GS activity: the slope of doublereciprocal plots increased with increasing concentrations of the inhibitor without change in $K_{\mathrm{m}}$.

The crude lyophilized fraction was also active on the GS of fungi other than $A$. fumigatus, including S. cerevisiae (E. Cabib, personal communication), Trichophyton rubrum and $T$. mentagrophytes (data not shown). Fraction 8-12 inhibited chitin synthase activity by $90 \%$ at a concentration similar to that which gave $100 \%$ inhibition of A. fumigatus GS. A GS-inhibitory (8-12) fraction was also isolated from protoplasts of two other species of Entomophthorales: Entomophthora muscae and Entomophaga aulicae.

GC analysis of the inhibitory fraction 8-12 from Entomophtbora schizophorae revealed that the sample contained approximately $32 \mu \mathrm{g}$ of FAs. The four major FAs and their relative composition were: palmitic acid, 16:0 $(32 \%)$; stearic acid, 18:0 (15\%); oleic acid, 18:1 $\omega 9$ $(43 \%)$; and linoleic acid, 18:2 $\omega 6(10 \%)$. Other longchain polyunsaturated $F A s$ were also present in trace amounts. Electrospray ionization confirmed this result and revealed the presence of other compounds, including 

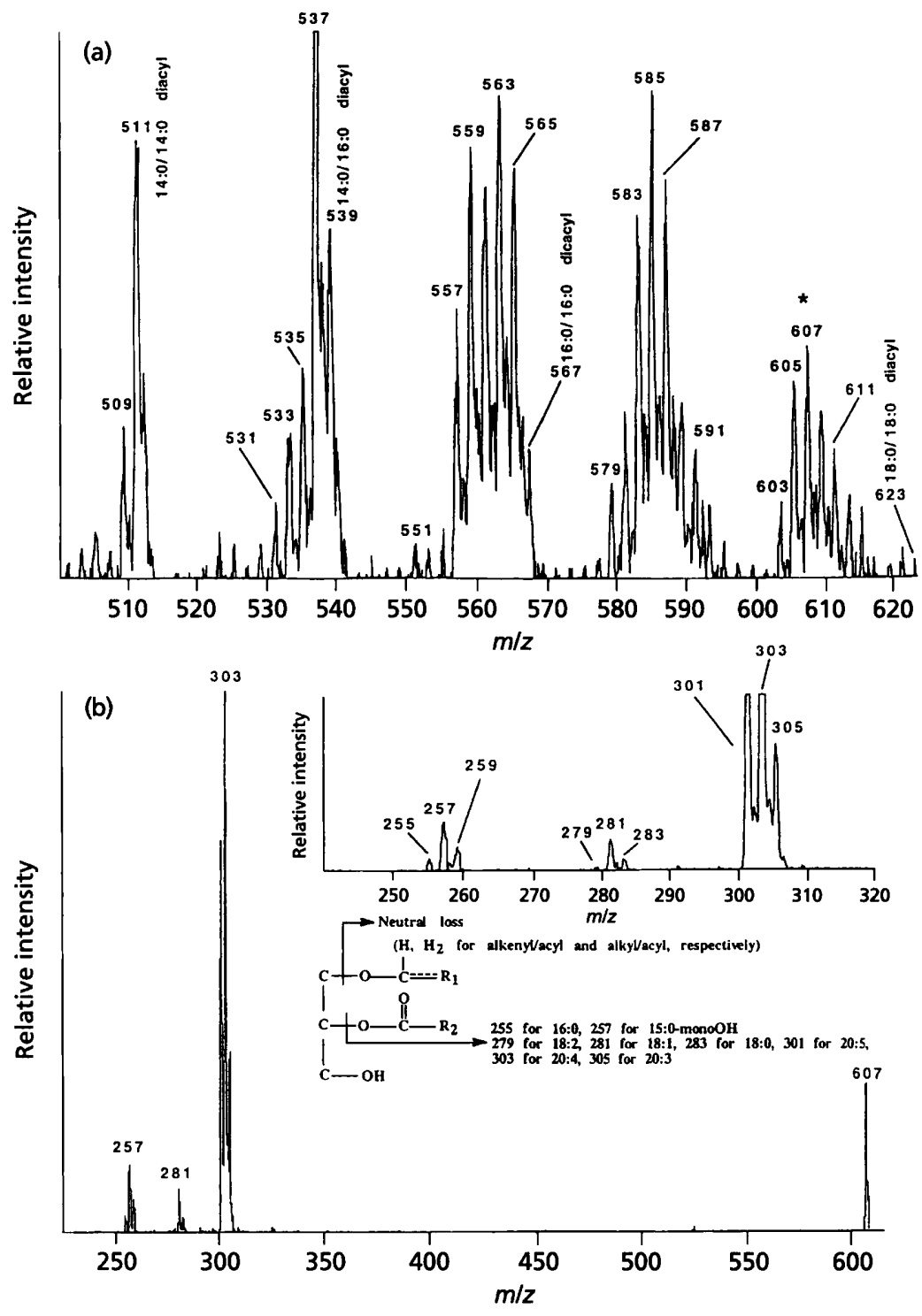

Fig. 2. (a) Negative ion electrospray MS spectrum of Entomophthora schizophorae DGs, with representative peaks marked. Note that many of these peaks consist of two to eight different DG molecular species. (b) Negative ion electrospray MS/MS spectrum of the peak at $\mathrm{m} / \mathrm{z} 607\left(^{*}\right)$ in (a). This peak is due to the synthesis of at least eight different ether-linked molecular species: 16:1/20:3 alkenyl/acyl, 16:1/20:4 alkyl/acyl, 16:0/20:5 alkyl/acyl, 18:4/18:0 alkenyl/acyl, 18:3/18:1 alkenyl/acyl, 18:2/18:2 alkenyl/acyl, 20:2/16:0 alkenyl/ acyl and 20:2/mono-OH-15:0 alkyl/acyl moieties. Species are reported as $[s n-1 / s n-2$ bond type], with the ether-linked moieties assumed to be in the $s n-1$ positions. The ether-linked (alkyl and alkenyl) FAs are not seen in the spectrum because they are lost as neutral species (Kerwin et al., 1994). Their identity is obtained by subtracting the mass of the FA in the $s n-2$ position from the parent ion.

several modified DGs (Fig. 2). An unexpected observation was the presence, in addition to the expected diacyl-linked DGs, of a very large amount of alkyl/acyl and alkenyl/ acyl DG moieties (Fig. 2b and unpublished observations). Fig. 2 also demonstrates the versatility of electrospray MS and MS/MS, in which a complex mixture of incompletely purified compouds can be rapidly and definitively identified using picogram or nanogram quantities of material.

\section{Inhibitory activity of FA}

Dipalmitin, distearin and diolein did not have significant inhibitory activity, nor did the saturated FAs palmitic acid $(16: 0)$ and stearic acid $(18: 0)$. The shorter chain myristic acid (14:0) was more active, with $67 \%$ inhibition at $0.33 \mathrm{~g}$ $1^{-1}$. The unsaturated $C_{18}$ FAs, however, including oleic acid (18:1), linoleic acid (18:2), linolenic acid (18:3), and $6,9,12,15$-octadecatetraenoic acid $(18: 4)$, at a concentration of $0.33 \mathrm{~g} \mathrm{l}^{-1}$, caused $90-100 \%$ inhibition. These results suggest that either a short chain, or a long unsaturated chain, is necessary for inhibition. Inhibition was much less, however, when the unsaturated FAs were tested at lower concentrations. None of them gave any inhibition at 3.33 or $0.33 \mathrm{mg} \mathrm{l}^{-1}$. Oleic acid gave $16 \%$ inhibition at $33 \mathrm{mg} \mathrm{l}^{-1}$, while 18:2,18:3 and 18:4 failed to give any significant inhibition at any of the lower concentrations. The concentration of $18: 1$ at $0.33 \mathrm{~g} \mathrm{l}^{-1}$, i.e $10 \mu \mathrm{g}$ per assay, is approximately equal to the amount of 18:1 found in the inhibitory HPLC fraction by GC.

\section{Effect of BSA on inhibitory activity}

At $1.5 \mathrm{~g}^{-1}$ BSA was found to have a protective effect against inhibition (Fig. 3). Greater amounts of BSA added to either the reaction mixtures with the protoplast inhibitor $\left(\mathrm{CHCl}_{3}\right.$ fraction) or the 18:1 FA standard resulted in lower percentages of inhibition. Increasing concentrations of BSA added to the assay with no added inhibitor resulted in an activation of GS. This suggests that the BSA was interacting with the protoplast inhibitor and the 18:1 FA standard, as well as with FAs present in the solubilized GS extract. 


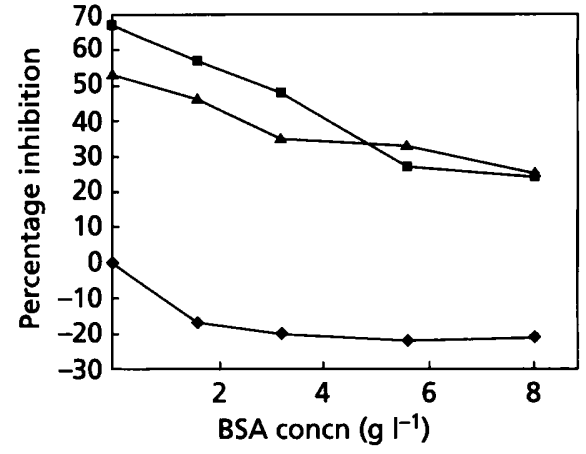

Fig. 3. Effect of $B S A$ on inhibition of $\mathrm{GS}$ activity by $\mathrm{CHCl}_{3}$ fraction ( $\boldsymbol{U}$ ) and by the 18:1 FA standard ( $\Delta$ ). Percentage inhibition decreased with increasing concentration of BSA. Activity increased (negative inhibition) when BSA was added to the reaction mixture without inhibitor $(\diamond)$.

\section{Effect of treatment with commercial lipase on inhibitory activity}

Inhibitory activity did not significantly increase with lipase treatment of the least purified $\mathrm{CHCl}_{3}$ fraction. Treatment of the 4:1 chloroform/methanol fraction resulted in an increase of inhibitory activity from $64 \%$ to $93 \%$.

Lipase treatment had different effects on the three pooled HPLC fractions eluted between 3 and 6 min (fractions 6-7, 8-9, 10-12). Fraction 8-9, consisting of both DGs and free FAs, had inhibitory activity that was unaffected by lipase treatment. The inhibitory activity of the 10-12 fraction, which consisted only of FAs, was also unaffected by lipase treatment. The 6-7 fraction, however, consisting mainly of DGs, inhibited only $3.5 \%$ without lipase treatment, but $34.5 \%$ after lipase treatment. This was accompanied by a change on the TLC plate: a diminution of the DG spots and the appearance of a FA spot. The effect of commercial DGs when treated with a lipase was similar. DGs by themselves gave no inhibition of GS, but after lipase treatment they gave $98 \%$ inhibition. The TLC also showed the disappearance of DGs and the appearance of a spot with an $R_{F}$ value corresponding to that of FAs. This high level of inhibition was due to free FAs. The presence of DGs in the 6-7 fraction from protoplasts after lipase treatment compared to commercial DGs, similarly treated, indicated that protoplast DGs were modified and less sensitive to lipase. This observation was confirmed by electrospray MS and MS/MS, and may be due to the high percentage of etherlinked DGs synthesized by the fungus. Treatment with boiled enzyme gave the same results as no lipase treatment. The lipase alone, with no substrate, caused no inhibition.

Extensive mass spectrometric examination of the inhibitory fractions, while confirming the presence of DGs and FAs, did not reveal any lipopeptides or glycopeptides similar to the previously documented GS inhibitors aculeacin, papulacandin and echinocandin (Iwata et al., 1982; Kang et al., 1986; Sawistowska-Shröder et al., 1984).

\section{DISCUSSION}

Unsaturated long-chain FAs present in the extract from Entomophthora schizophorae protoplasts were responsible for the inhibition of GS. Among all FAs present in this extract, oleic acid was the most potent inhibitor. Amounts of standard unsaturated FAs comparable to those found in the purified fractions gave very high inhibition, making it unlikely that inhibition was caused by other compounds present in the purified fraction. Protoplast extracts which were not incubated at $37^{\circ} \mathrm{C}$ were found to contain DGs but neither FAs nor inhibitory activity. This result suggests that the FAs are generated by the action of one or more lipases in the protoplast extract when incubated for $36 \mathrm{~h}$ at $37^{\circ} \mathrm{C}$. FAs were also found to be active against the chitin synthase of $A$. fumigatus. Since chitin synthase of $A$. fumigatus or GS of T. rubrum and T. mentagrophytes were not solubilized by CHAPS as was GS of $A$. fumigatus, the action of FAs on GS was not due to a disruption of micelles formed by CHAPS. Instead, FAs presumably, by distupting the membrane equilibrium, affected the activity of the polysaccharide synthases, which are multi-enzyme complexes. The lack of inhibition at low concentrations of FAs supports the idea that the mechanism of inhibition may involve disruption of the membrane, for which relatively large concentrations of FAs are necessary.

Although an inhibitor of GS can be purified from entomophthoralean protoplasts, it is not a novel or unique compound. Many of the antifungal drugs targeted against GS, including cilofungin and papulacandin B, contain FA side-chains. These compounds have been shown to be ineffective without side-chains (Taft \& Seletrennikoff, 1990; Varona et al., 1983). It has also been shown that FAs and monoglycerides cause a concentration-dependent decrease in the activity of yeast GS due to minor perturbations of the plasma membrane (Ko et al., 1994). Likewise, DGs, which do not inhibit GS, are unable to alter membrane fluidity (Ko et al., 1994). Maresca et al. (1994) also showed that desaturation of membrane lipids allowed a change in the morphogenesis of Histoplasma. Our results, that long-chain unsaturated free FAs, at relatively high concentrations, are responsible for inhibition, are consistent with these previous observations.

The unsaturated FAs cannot be the sole cause of the complete inhibition of the GS activity in protoplasts, since similar inhibitory activity was found in a (8-12) fraction isolated from hyphal body extract of Entomophaga aulicae (data not shown). This result suggests that the inhibitory activity due to FA is not specific. While free FAs by themselves offer little hope for the development of a pharmaceutical antifungal agent, it is possible that any existing drug compounds that rely upon FA side-chains for activity could be made more effective with a longchain unsaturated FA side-chain.

\section{ACKNOWLEDGEMENTS}

This work was supported by a grant from Roussel-Uclaf to J. P. Latgé and a grant from the National Institutes of Health (5 R01 AI22993) to J. Kerwin. 


\section{REFERENCES}

Beaulieu, D., Tang, J., Zeckner, D. \& Parr, T. (1993). Correlation of cilofungin in vivo efficacy with its activity against Aspergillus fumigatus (1,3)- $\beta$-D-glucan synthase. FEMS Microbiol Lett 108, 133-138.

Beauvais, A. \& Latgé, J. P. (1988). A simple medium to grow Entomophthoralean protoplasts. J Invertebr Pathol 51, 175-178.

Beauvais, A. \& Latgé, J. P. (1989). Chitin and $\beta(1-3)$ glucan synthases in the protoplastic entomophthorales. Arch Microbiol 152, 229-236.

Beauvais, A., Latgé, J. P., Vey, A. \& Prevost, M. C. (1989). The role of surface components of the entomopathogenic fungus Entomophaga aulicae in the cellular immune response of Galleria mellonella (Lepidoptera). J Gen Microbiol 135, 489-498.

Beauvais, A., Drake, R., Ng, K., Diaquin, M. \& Latgé, J. P. (1993). Characterization of the $1,3-\beta$-glucan synthase of Aspergillus fumigatus. J Gen Microbiol 139, 3071-3078.

Cabib, E., Bowers, B., Sburlati, A. \& Silverman, S. J. (1988). Fungal cell wall synthesis: the construction of a biological structure. Microbiol Sci 5, 370-375.

Fèvre, M. (1979). Glucanases, glucan synthases and wall growth in Saprolegnia monoica. In Fungal Walls and Hyphal Growth, pp. 225-263. Edited by J. H. Burnett \& A. P. J. Trinci. Cambridge: Cambridge University Press.

Fromtling, R. \& Abruzzo, G. (1989). L-671,329, a new antifungal agent: in vitro activity, toxicity and efficacy in comparison to aculeacin. $J$ Antibiot 42, 174-178.

Hamilton, J., Era, S., Bhamidipati, S. \& Reed, R. (1991). Locations of the three primary binding sites for long-chain fatty acids on bovine serum albumin. Proc Natl Acad Sci US A 88, 2051-2054.

Higgins, J. A. (1987). Separation and analysis of membrane lipid components. In Biological Membranes: a Practical Approach, pp. 103-137. Edited by J. B. C. Findlay \& W. H. Evans. Oxford: IRL Press.
Iwata, K., Yamamoto, Y., Yamaguchi, H. \& Hiratani, T. (1982). In vitro studies of Aculeacin A, a new antifungal antibiotic. $J$ Antibiot 35, 203-209.

Kang, M. S., Szaniszlo, P. J., Notario, V. \& Cabib, E. (1986). The effect of Papulacandin B on (1-3)- $\beta$-D-glucan synthetases. A possible relationship between inhibition and enzyme conformation. Carbobydr Res 149, 13-21.

Kates, M. (editor) (1986). Techniques of Lipidology: Isolation, Analysis and Identification of Lipids. Amsterdam: Elsevier

Kerwin, J. L., Tuininga, A. R. \& Ericsson, L. H. (1994). Identification of molecular species of glycerophospholipids and sphingomyelin using electrospray mass spectrometry. $J$ Lipid Res 35, 1102-1114.

Ko, Y. T., Frost, D., Ho, C. T., Ludescher, R. \& Wasserman, B. (1993). Inhibition of yeast (1,3)- $\beta$-glucan synthase by phospholipase $\mathrm{A}_{2}$ and its reaction products. Biochim Biophys Acta 1193, 31-40.

Maresca, B., Carratu, L. \& Kobayashi, G. S. (1994). Morphological transition in the human fungal pathogen Histoplasma capsulatum. Trends Microbiol 2, 110-114.

Sawistowska-Schroder, E. T., Kerridge, D. \& Perry, H. (1984). Echinocandin inhibition of 1,3- $\beta$-D-glucan synthase from Candida albicans. FEBS Lett 173, 134-138.

Taft, C. S. \& Seletrennikoff, C. P. (1990). Cilofungin inhibition of (1-3)- $\beta$-glucan synthase: the lipophilic side chain is essential for inhibition of enzyme activity. $J$ Antibiot 43, 433-437.

Thomsen, L. \& Beauvais, A. (1995). Cloning of two chitin synthase gene fragments from a protoplastic entomophthorale. FEMS Microbiol Lett 129, 115-120.

Varona, R., Perez, P. \& Duran, A. (1983). Effect of papulacandin B on $\beta$-glucan synthesis in Sshzosaccharomyces pombe. FEMS Microbiol Lett 20, 243-247.

Received 12 May 1995; accepted 27 June 1995. 